\title{
The ATLAS Forward Physics Project
}

\author{
Christophe Royon
}

\begin{abstract}
We describe the main components of the ATLAS Forward Physics project, namely the movable beam pipe, the tracking and timing detectors which allow to detect intact protons in the final state at the LHC. The position detector is composed on 6 layers of 3D silicon detectors readout by FE-I4 chips developped for ATLAS. The fast timing detector is built from a quartz-based Cerenkov detector coupled to a microchannel plate photomultiplier tube, followed by the electronic elements that amplify, measure, and record the time of the event along with a stabilized reference clock signal, ensuring a time resolution of 10-15 picoseconds.
\end{abstract}

We describe the proposal to install the ATLAS Forward Proton (AFP) detector in order to detect intact protons at 206 and 214 meters on both side of the ATLAS experiment [1]. This one arm will consist of two sections (AFP1 and AFP2) contained in a special design of beampipe described in Section I. In the first section (AFP1), in one pocket of the beampipe, a tracking station composed by 6 layers of Silicon detectors described in Section II will be deployed. The second station AFP2 will contains another tracking station similar to the one already described and a timing detector described in Section III. The aim of this setup, mirrored by an identical arm placed on the opposite side of the ATLAS IP, will be to tag the protons emerging intact from the $p p$ interactions so allowing ATLAS to exploit the program of diffractive and photoproduction processes [2]. This device is sensitive to diffractive masses in the ATLAS detectors between 350 and $1.4 \mathrm{TeV}$. The advantage of this apparatus from the physics point of view is that the system is fully constrained: we measure all the particles in the final state, namely the two intact protons and the particle produced ( $W, Z$, the Higgs boson or the SUSY particles). The mass and the kinematical properties of the produced particles can be computed using the information on the tagged protons.

\section{Movable BeAm PIPES}

The idea of movable Hamburg beam pipes is quite simple [3]: a larger section of the LHC beam pipe than the usual one can move close to the beam using bellows so that the detectors located at its edge (called pocket) can move close to the beam by about $2.5 \mathrm{~cm}$ when the beam is stable (during injection, the detectors are in parking position). In its design, the predominant aspect is the minimization of the thickness of the portions called floor and window (see Fig. 11). Minimizing the depth of the floor ensures that the detector can go as close to the beam as possible allowing us to detect protons scattered at very small angles, while minimizing the depth of the thin window is important to keep the protons intact and to reduce the impact of multiple interactions. Two configurations exist for the movable beam pipes: the first one at $206 \mathrm{~m}$ form the ATLAS interaction point hosts a Si detector (floor length of

DSM/IRFU/SPP, CEA Saclay, France; On behalf of the AFP collaboration

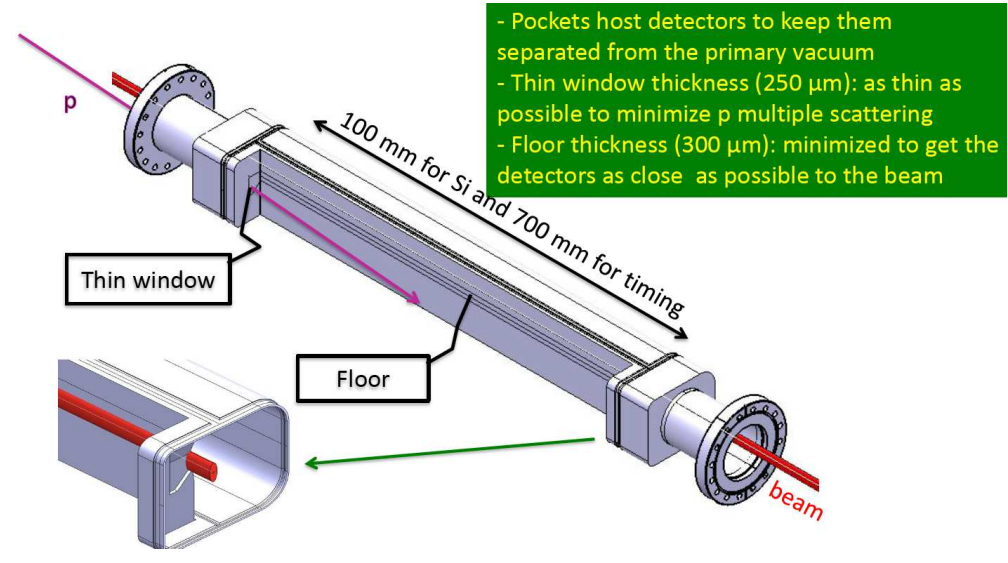

Fig. 1. Scheme of the movable beam pipe.

about $100 \mathrm{~mm}$ ) and the second one (floor length of about 700 $\mathrm{mm}$ ) the timing and the Si detectors.

\section{3D Silicon DETECTORS}

The purpose of the tracker system is to measure points along the trajectory of beam protons that are deflected at small angles as a result of collisions. The tracker when combined with the LHC dipole and quadrupole magnets, forms a powerful momentum spectrometer. Silicon tracker stations will be installed in Hamburg beam pipes (HBP) at \pm 206 and $\pm 214 \mathrm{~m}$ from the ATLAS.

The key requirements for the silicon tracking system at $220 \mathrm{~m}$ are:

- Spatial resolution of $\sim 10$ (30) $\mu \mathrm{m}$ per detector station in $x(y)$

- Angular resolution for a pair of detectors of about $1 \mu \mathrm{rad}$

- High efficiency over the area of $20 \mathrm{~mm} \times 20 \mathrm{~mm}$ corresponding to the distribution of diffracted protons

- Minimal dead space at the edge of the sensors allowing us to measure the scattered protons at low angles

- Sufficient radiation hardness in order to sustain the radiation at high luminosity

- Capable of robust and reliable operation at high LHC luminosity

The basic building unit of the AFP detection system is a module consisting of an assembly of a sensor array, onsensor read-out chip(s), electrical services, data acquisition and detector control system. The module will be mounted on the mechanical support with embedded cooling and other necessary services. The sensors are double sided 3D $50 \times 250$ micron pixel detectors with slim-edge dicing built by FBK and CNM. The sensor efficiency has been measured to be close to $100 \%$ over the full size in beam tests. A possible upgrade of this device will be to use 3D edgless Silicon detectors 
built in a collaboration between SLAC, Manchester, Oslo, Bergen... A new front-end chip FE-I4 has been developed for the Si detector by the Insertable B Layer (IBL) collaboration in ATLAS. The FE-I4 integrated circuit contains readout circuitry for 26880 hybrid pixels arranged in 80 columns on $250 \mu \mathrm{m}$ pitch by 336 rows on $50 \mu \mathrm{m}$ pitch, and covers an area of about $19 \mathrm{~mm} \times 20 \mathrm{~mm}$. It is designed in a $130 \mathrm{~nm}$ feature size bulk CMOS process. Sensors must be DC coupled to FE-I4 with negative charge collection. The FE-I4 is very well suited to the AFP requirements: the granularity of cells provides a sufficient spatial resolution, the chip is radiation hard enough (up to a dose of $3 \mathrm{MGy}$ ), and the size of the chip is sufficiently large that one module can be served by just one chip.

The dimensions of the individual cells in the FE-I4 chip are $50 \mu \mathrm{m} \times 250 \mu \mathrm{m}$ in the $x$ and $y$ directions, respectively. Therefore to achieve the required position resolution in the $x$ direction of $\sim 10 \mu \mathrm{m}$, six layers with sensors are required (this gives $50 / \sqrt{12} / \sqrt{5} \sim 7 \mu \mathrm{m}$ in $x$ and roughly 5 times worse in $y$ ). Offsetting planes alternately to the left and right by one half pixel will give a further reduction in resolution of at least $30 \%$. The AFP sensors are expected to be exposed to a dose of $30 \mathrm{kGy}$ per year at the full LHC luminosity of $10^{34} \mathrm{~cm}^{-2} \mathrm{~s}^{-1}$.

\section{TIMING DETECTORS}

A fast timing system that can precisely measure the time difference between outgoing scattered protons is a key component of the AFP detector. The time difference is equivalent to a constraint on the event vertex, thus the AFP timing detector can be used to reject overlap background by establishing that the two scattered protons did not originate from the same vertex as the the central system. The final timing system should have the following characteristics [5]:

- $10 \mathrm{ps}$ or better resolution (which leads to a factor 40 rejection on pile up background)

- Efficiency close to $100 \%$ over the full detector coverage

- High rate capability (there is a bunch crossing every 25 ns at the nominal LHC)

- Enough segmentation for multi-protin timing

- Level trigger capability

Figure 2 shows a schematic overview of the first proposed timing system, consisting of a quartz-based Cerenkov detector coupled to a microchannel plate photomultiplier tube (MCPPMT), followed by the electronic elements that amplify, measure, and record the time of the event along with a stabilized reference clock signal. The QUARTIC detector consists of an array of $8 \times 4$ fused silica bars ranging in length from about 8 to $12 \mathrm{~cm}$ and oriented at the average Cerenkov angle. A proton that is sufficiently deflected from the beam axis will pass through a row of eight bars emitting Cerenkov photons providing an overall time resolution that is approximately $\sqrt{8}$ times smaller than the single bar resolution of about $30 \mathrm{ps}$, thus approaching the $10 \mathrm{ps}$ resolution goal. Prototype tests have generally been performed on one row ( 8 channels) of 5 $\mathrm{mm} \times 5 \mathrm{~mm}$ pixels, while the initial detector is foreseen to have four rows to obtain full acceptance out to $20 \mathrm{~mm}$ from the beam. The beam tests lead to a time resolution per bar of the order of $34 \mathrm{ps}$. The different componenets of the timing

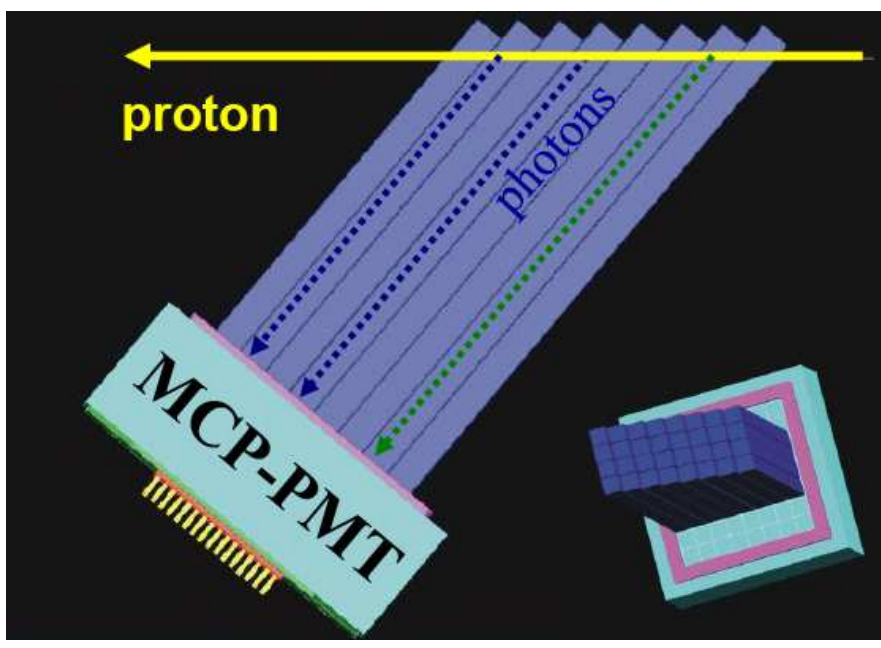

Fig. 2. A schematic diagram of the QUARTIC fast timing detector.

\begin{tabular}{|l|c|c|l|c|}
\hline \multicolumn{1}{|c|}{ Component } & $\begin{array}{c}\delta t(p s) \\
\text { Current }\end{array}$ & $\begin{array}{c}\delta \text { t(ps) } \\
\text { Projected } \\
(8 \text { ch +cable) }\end{array}$ & $\begin{array}{l}\text { Improve } \\
\text { ment }\end{array}$ & $\begin{array}{c}\delta t(p s) \\
\text { Phase 0 } \\
\text { (8 channels) }\end{array}$ \\
\hline $\begin{array}{l}\text { Radiator (fused silica bar) } \\
\text { 10 pe's }\end{array}$ & 22 & 22 & $\begin{array}{l}\text { Optimize } \\
\text { radiator }\end{array}$ & 17 \\
\hline $\begin{array}{l}\text { MCP-PMT (64 channel 25 } \\
\text { um Planacon) }\end{array}$ & 20 & 20 & 10 um tube & 15 \\
\hline CFD & 5 & 5 & - & 5 \\
\hline HPTDC & 16 & 16 & - & 15 \\
\hline Reference Clock & - & 3 & - & 3 \\
\hline Total/bar & 34 & 34 & & 28 \\
\hline Cable & 14 & 14 & - & $5 \%$ \\
\hline Total/ detector & & $15 \%$ & retune CFD & 10 \\
\hline
\end{tabular}

Fig. 3. Different components of the timing detector resolution.

resolution are given in Fig. 3. The upgraded design of the timing detector has equal rate pixels, and we plan to reduce the the width of detector bins close to the beam, where the proton density is highest.

At higher luminosity of the LHC (phase I starting in 2019), higher pixelisation of the timing detector will be required. For this sake, a R\&D phase concerning timing detector developments based on SiPMs, APDs, quartz fibers, diamonds has been started in Saclay. In parallel, a new timing readout chip has been developed. It uses waveform sampling methods which give the best possible timing resolution. The aim of this chip called SAMPIC [6] is to obtain sub $10 \mathrm{p}$ timing resolution, $1 \mathrm{GHz}$ input bandwidth, no dead time at the LHC, and data taking at 2 Gigasamples per second. The cost per channel is estimated to be of the order for $10 \$$ which a considerable improvement to the present cost of 10,000 \$ per channel, allowing us to use this chip in medical applications such as PET imaging detectors. The holy grail of imaging 10 picosecond PET detector seems now to be feasible: with a resolution better than $20 \mathrm{ps}$, image reconstruction is no longer necessary and real-time image formation becomes possible [7].

\section{REFERENCES}

[1] ATLAS Coll., CERN-LHCC-2011-012. 
[2] E. Chapon, O. Kepka, C.Royon, Phys. Rev. D81 (2010) 074003; M. Boonekamp, F. Chevallier, C. Royon. Acta Phys.Polon. B40 (2009) 22392321.

[3] K. Piotrzkowski, U. Schneekloth, Proc. of the ZEUS Collaboration meeting, March 1994, DESY, Hamburg.

[4] ATLAS IBL Coll., CERN-LHCC-2010-013, ATLAS-TDR-019 5/09/2010.

[5] A. Brandt, Microchannel Plate PMT Lifetime and Performance, DIRC2011: Workshop on Fast Cerenkov detectors, Giessen, Germany, April 4-6, 2011.

[6] E. Delagnes, D. Breton, F. Lugiez, R. Rahmanifard, Nucl. Science IEEE Transactions, Volume 54, Issue 5, Part 2, Oct 2007, 1735; E. Delagnes, patent WO 2008/050177, EP2076963.

[7] M. Dosanjh, talk at the ATLAS collaboration meeting, October 1-5 2012, Montreux, Switzerland. 